\title{
USO DE ÍNDICE DE VEGETAÇÃO NA ESTIMATIVA DA PRODUÇÃO DE BIOMASSA DE PLANTAS DE COBERTURAS DO SOLO
}

Diego Armando Amaro da Silva ${ }^{1}$, Antônio Luis Santi ${ }^{2}$, Mauricio Siqueira dos Santos ${ }^{3}$; Romano Augusto Martini Dal Bello ${ }^{3}$, Renan Tonin Martini ${ }^{3}$

1 Engo Agro Mestrando do Programa de Pós-graduação em Agronomia - Agricultura e Ambiente da Universidade Federal de Santa Maria, Bolsista Capes (damaro.agro@gmail.com), Frederico Westphalen, RS, Brasil.

2 Eng $^{\circ}$ Agr ${ }^{\circ}$ Professor Doutor da Universidade Federal de Santa Maria, Frederico Westphalen, Brasil.

3 Graduandos do curso de Agronomia da Universidade Federal de Santa Maria, Frederico Westphalen, Brasil.

Recebido em: 08/09/2015 - Aprovado em: 14/11/2015 - Publicado em: 01/12/2015 DOI: http://dx.doi.org/10.18677/Enciclopedia_Biosfera_2015_241

\section{RESUMO}

Avanços tecnológicos na agropecuária têm mostrado a importância de se medir a variabilidade espacial dos atributos do solo e de planta que afetam ou determinam o rendimento das lavouras, fornecendo uma ferramenta valiosa que não estava disponível anteriormente, permitindo interpretar situações semelhantes de forma diferenciada. Objetivou-se avaliar a eficiência do índice de vegetação por diferença normalizada (NDVI) para estimar a fitomassa seca produzida por dez sistemas de plantas de cobertura do solo no norte do Estado do Rio Grande do Sul. Foram estabelecidos coeficientes de determinação $\left(R^{2}\right)$ entre o NDVI observado e a matéria seca da biomassa coletada nos sistemas de plantas de cobertura do solo com 10 repetições. Os dez sistemas utilizados foram: aveia preta (AP), aveia branca (AB), azevém (AZ), nabo forrageiro (NF), ervilhaca $(E)$, consórcio $A P+N F+E$, consórcio $A P+N F$, consórcio $A P+E$, tremoço branco $(T B)$, vegetação espontânea ou pousio (P). O NDVI foi capaz de estimar a massa da matéria seca de forma satisfatória em sete sistemas analisados, mostrando ser uma ferramenta valiosa também na entressafra. Os sistemas que estavam em floração tiveram o $R^{2}$ prejudicado. Não é recomendada a utilização do NDVI para estimar fitomassa seca em consórcios envolvendo três ou mais plantas, ou analisar os dados de diferentes plantas de cobertura em conjunto, por se mostrar pouco eficiente nestes casos.

PALAVRAS-CHAVE: agricultura de precisão, GreenSeeker, NDVI, palhada.

\section{USE OF VEGETATION INDEX IN ESTIMATE BIOMASS PRODUCTION BY COVER CROPS}

\begin{abstract}
Technological advances in agriculture have shown the importance of measuring the spatial variability of soil properties and plant that affect or determine crop yields, providing a valuable tool that was not previously available, allowing interpret similar
\end{abstract}


situations differently. The objective of this study was to evaluate the efficiency of normalized difference vegetation index (NDVI) to estimate the dry matter produced for ten systems of ground cover plants in the north of the Rio Grande do Sul State. Were established determination coefficients $\left(R^{2}\right)$ between the observed NDVI and dry biomass collected of ten cover crops systems with ten repetitions each. Determination coefficients were established $\left(R^{2}\right)$ between the observed NDVI and dry biomass collected ten of soil cover crops systems with 10 repetitions each. The ten systems used were: black oat $(A P)$, oat $(A B)$, ryegrass $(A Z)$, radish $(N F)$, vetch $(E)$, consortium $A P+N F+E$, consortium $A P+N F$, consortium $A P+E$, white lupine $(T B)$, and spontaneous vegetation or fallow $(\mathrm{P})$. The NDVI was able to estimate the dry matter satisfactorily in seven systems analyzed, proving to be a valuable tool also in the off season. Systems that were in bloom had harmed $R^{2}$. It is not recommended to use the NDVI to estimate biomass in consortiums involving three or more plants, or analyze data from different plant cover together, for being inefficient in these cases.

KEYWORDS: precision agriculture, GreenSeeker, NDVI, straw.

\section{INTRODUÇÃO}

O uso de plantas de cobertura (PC), que é uma prática antiga como forma de melhorar o potencial produtivo das áreas utilizadas na agricultura (ZIECH et al., 2014), vem ganhando cada vez mais espaço no Sistema de Plantio Direto (SPD) nas áreas agrícolas do Sul do Brasil, por atender uma das premissas básicas do SPD, que é a adequação de sistemas de rotação e sucessão de culturas de modo a aumentar o aporte de material orgânico e nutrientes, bem como proteger o solo dos processos erosivos (MARCELO et al., 2009), sendo fundamental selecionar PCs com maior potencial em produzir fitomassa e acumular principalmente, $\mathrm{C}$ e $\mathrm{N}$ contidos em seus tecidos (DONEDA et al., 2012).

A produção de matéria seca das espécies utilizadas para cobertura do solo depende de inúmeras condições, como as edafoclimáticas, fitossanitárias, de práticas de manejo, e em grande parte, da agressividade do sistema radicular (CARVALHO \& AMABILE, 2006; CARVALHO et al., 2013), sendo essencial a criação de uma ferramenta adequada para estimar a produção desta em tempo real.

$\mathrm{Na}$ agricultura de precisão, utilizam-se de forma intensa, as geotecnologias, tais como sensoriamento remoto, tais como obter informações de objetos, sem contato físico, por meio de uso de sensores (DEGHAID et al., 2013), e nos últimos anos, têm ganhado destaque os estudos sobre parametrização de atributos morfológicos dos dosséis vegetais, feitos com métodos baseados nas propriedades ópticas das plantas (OLLINGER, 2011; SIMÕES et al., 2015).

Diversos índices gerados pela combinação de valores de refletância entre bandas espectrais específicas vêm sendo utilizados, como indicadores da quantidade de vegetação e outros atributos dos dosséis, sendo o Índice de Vegetação por Diferença Normalizada (NDVI), o mais conhecido (OLLINGER, 2011; SIMÕES et al., 2015), sendo que o NDVI apresenta ótima correlação linear com a biomassa da vegetação em casos que o terreno apresente boa cobertura vegetal (MENESES \& ALMEIDA, 2012; DEGHAID et al., 2013), assim como as plantas de cobertura do solo.

Dessa forma, o objetivo deste trabalho foi avaliar a eficiência do NDVI para estimar a fitomassa seca produzida por dez sistemas de plantas de cobertura do solo diferentes no norte do Estado do Rio Grande do Sul. 


\section{MATERIAL E MÉTODOS}

O experimento foi conduzido no município de Frederico Westphalen - RS na safra agrícola de 2014, na área experimental da Universidade Federal de Santa Maria, campus de Frederico Westphalen - RS, localizada a 27023'24.33" S e 5325'39.58" O, com altitude de $483 \mathrm{~m}$. Segundo a classificação climática de Köppen, o clima da região é Cfa ou subtropical úmido, com temperatura média anual de $19^{\circ} \mathrm{C}$ e precipitação entre 1800 e $2000 \mathrm{~mm}$ bem distribuídos ao longo do ano.

O solo foi classificado conforme a EMBRAPA (2013) como Latossolo Vermelho distrófico típico, com textura argilosa, cujas características físicas e químicas do solo de 0,00-0,20 m de profundidade se encontram na Tabela 1.

TABELA 1. Composição e características químicas e físicas do solo da área experimental.

\begin{tabular}{lcc}
\hline Análise & Unidade & Valor \\
\hline $\mathrm{pH}$ em água & $-\log \left[\mathrm{H}^{+}\right]$ & 5,1 \\
Argila & $\mathrm{g} \mathrm{kg}^{-1}$ & 740 \\
Matéria orgânica (MOS) & $\mathrm{g} \mathrm{kg}^{-1}$ & 32 \\
$\mathrm{P}\left(\right.$ Mehlich $\left.^{-1}\right)$ & $\mathrm{mg} \mathrm{dm}^{-3}$ & 9,3 \\
$\mathrm{~K}^{+}$ & $\mathrm{mg} \mathrm{dm}$ & 268,0 \\
$\mathrm{Al}^{3+}$ & $\mathrm{cmol}_{\mathrm{c}} \mathrm{dm}^{-3}$ & 0,4 \\
$\mathrm{Acidez} \mathrm{potencial}^{-3}$ & $\mathrm{cmol}_{\mathrm{c}} \mathrm{dm}^{-3}$ & 5,6 \\
$\mathrm{Ca}^{++}$ & $\mathrm{cmol}_{\mathrm{c}} \mathrm{dm}^{-3}$ & 6,0 \\
$\mathrm{Mg}^{++}$ & $\mathrm{cmol}_{\mathrm{c}} \mathrm{dm}^{-3}$ & 2,8 \\
Capacidade de Troca de Cátions (CTC) & $\mathrm{cmol}_{\mathrm{c}} \mathrm{dm}^{-3}$ & 11,3 \\
Saturação da CTC por bases (V\%) & $\%$ & 50,6 \\
\hline
\end{tabular}

Foram efetuadas as três leituras de NDVI em cada uma das dez parcelas por tratamento. Na mesma data foi feito o corte de $0,25 \mathrm{~m}^{2}(0,5 \times 0,5 \mathrm{~m})$ de biomassa vegeetal, que foram levadas a estufa com ventilação forçada á $65 \stackrel{\circ}{\circ} \mathrm{C}$ até peso constante para determinar a massa da matéria seca (MMS) de cada parcela. As plantas de cobertura foram semeadas na primeira quinzena do mês de maio de 2014, sendo as leituras de NDVI e coletas de MMS, efetuadas dois dias antes da dessecação do experimento (primeira semana de setembro) para permitir a semeadura da cultura da soja no local. Cada sistema de planta de cobertura de inverno possuía dimensões de $5 \mathrm{~m} \times 25 \mathrm{~m}$ cada, divididas em dez parcelas por tratamento $\left(5 \mathrm{~m} \times 2,5 \mathrm{~m}\right.$ cada) e área total de $1250 \mathrm{~m}^{2}$. Os dez sistemas de plantas de cobertura analisados foram: aveia preta (Avena strigosa S. - AP), aveia branca (Avena sativa L. - AB), azevém (Lolium multiflorum L. - AZ), nabo forrageiro (Raphanus sativus L. - NF), ervilhaca (Vicia sativa L. - E), aveia preta + ervilhaca + nabo forrageiro $(\mathrm{AP}+\mathrm{NF}+\mathrm{E})$, aveia preta + nabo forrageiro $(\mathrm{AP}+\mathrm{NF})$, aveia preta + ervilhaca $(\mathrm{AP}+\mathrm{E})$, tremoço branco (Lupinus albus L. - TB) e pousio (vegetação espontânea - P).

As leituras de NDVI foram realizadas com aparelho GreenSeeker® 505 Handheld Sensor, que calcula o NDVI a partir da equação $N D V l=(\rho i v p-\rho v) /(\rho i v p+\rho v)$, onde pivp é a refletância no infravermelho próximo $(0,725-1,1 \mu \mathrm{m})$, e $\rho v$ é a refletância no vermelho $(0,58-0,68 \mu \mathrm{m})$. Seus valores variam de -1 a 1 de modo que, quanto mais próximo de 1 maior o vigor de desenvolvimento da cultura (ROUSE et al., 1978; GROHS et al., 2011), ou maior a cobertura de solo 
pela mesma. A altura de leitura foi fixa segundo a recomendação do fabricante, com distância entre o topo do dossel e o sensor de aproximadamente 0,8 a $1 \mathrm{~m}$, sendo realizadas todas entre as 09 e 12 horas (CRUSIOL et al., 2013).

Os dados de fitomassa seca e NDVI foram analisados de acordo com o modelo de delineamento experimental inteiramente casualizado, submetidos à análise da variância pelo teste $\mathrm{F}(\mathrm{p}<0,05)$, e as médias comparadas pelo teste $t$ de Student $(p<0,05)$, conforme FERREIRA et al. (2012). Ainda foram feitas regressões para estimar o coeficiente de determinação de fitomassa seca da parcela através das leituras de NDVI. Para as análises dos dados foi utilizado o programa estatístico ASSISTAT, tomando como base os níveis de significância maiores que 95\% ( $p \leq$ $0,05)$.

\section{RESULTADOS E DISCUSSÃO}

Os valores obtidos de massa da matéria seca (MMS) foram classificados segundo PIMENTEL-GOMES (1985), onde se considera que quanto menor o CV\%, mais homogêneos são os dados. $A B$ e $A Z$ apresentaram baixa variabilidade $(C V \%=$ 9,6 e 7,5), e sendo detectada alta variabilidade em AP $(C V \%=22,3)$, provavelmente devido ao acamamento dessa cultura, muito alta variabilidade em $\mathrm{P}(\mathrm{CV} \%=59,6)$, e média variabilidade entre os demais tratamentos (CV\% entre 10,7 e 15,8).

Os tratamentos que produziram maior quantidade de MMS foram AP+E e TB, seguido de AP, como pode ser visto na Figura 1. Estes dados corroboram com os resultados encontrados por CARVALHO et al. (2013), destacando que os consorciamentos tendem a produzir uma quantidade maior de massa da matéria seca podendo proporcionar benefícios aos sistemas de produção de distintas formas, além de não recomentar a prática do pousio, visto que produz pouca massa da matéria fresca e possui uma menor cobertura vegetal.

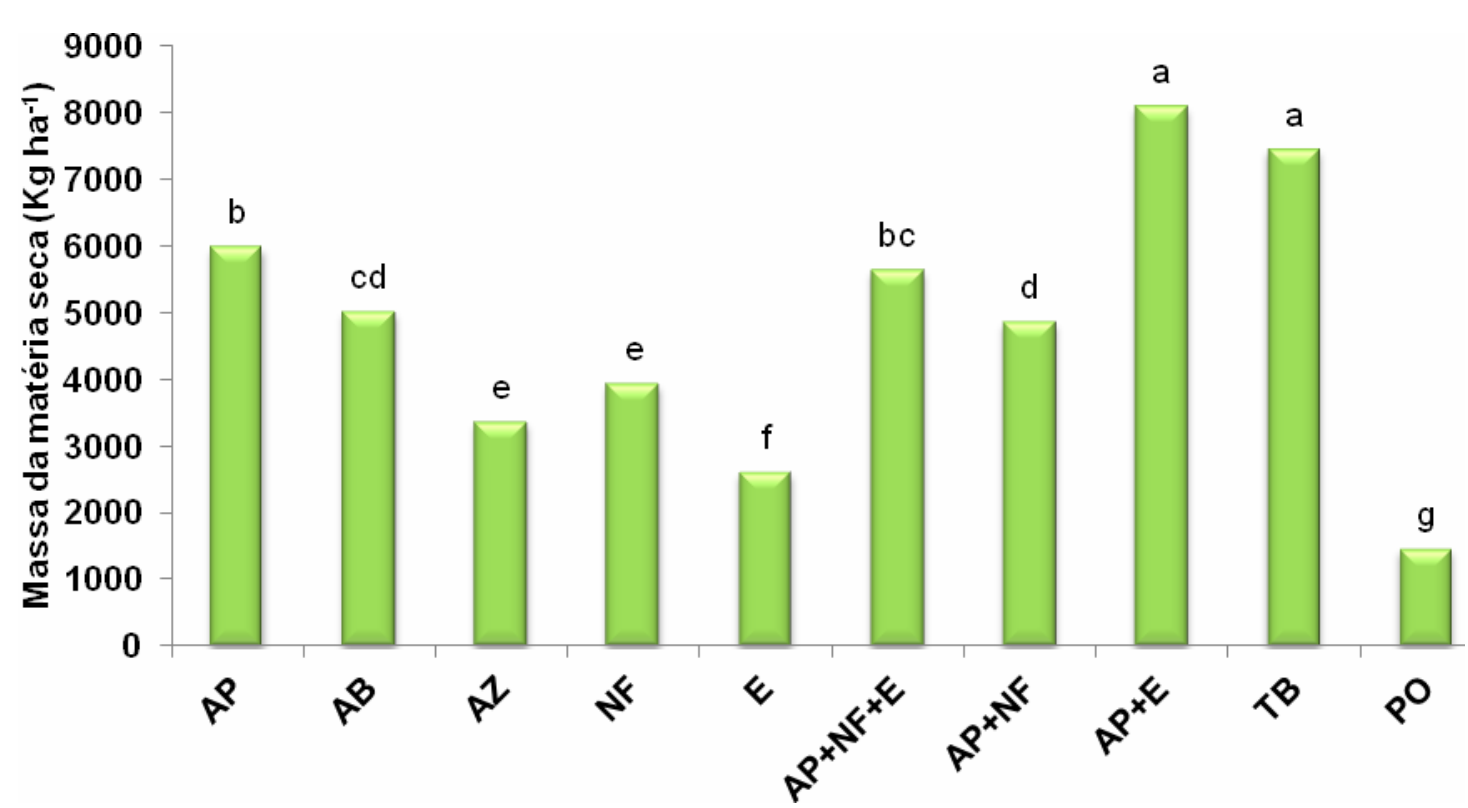

FIGURA 1. Produção de Massa da matéria seca em diferentes sistemas e plantas de cobertura do solo. Aveia preta (AP), aveia branca (AB), azevém (AZ), nabo forrageiro (NF), ervilhaca $(E)$, tremoço branco (TB), vegetação espontânea ou pousio (PO). Médias seguidas de mesma letra, não diferem pelo teste Tukey a $5 \%$ probabilidade. 
Já para os valores de NDVI (Figura 2), que apresentaram baixo CV com exceção do $P(12,2 \%)$, o tratamento $E$ mostrou o maior valor observado $(0,86)$, apesar de ser uma das PCs que menos produziram fitomassa seca. Isto demonstra que as PCs analisadas não devem ser interpretadas de forma conjunta, devendo-se apenas comparar os dados entre cada cobertura analisada. Ainda, RISSO et al. (2012) destaca que uma das peculiaridades inerentes ao NDVI é a saturação assintórica, que torna o índice incapaz de diferenciar biomassa verde em condições de elevado IAF e cobertura do solo, atendido pelo tratamento citado.

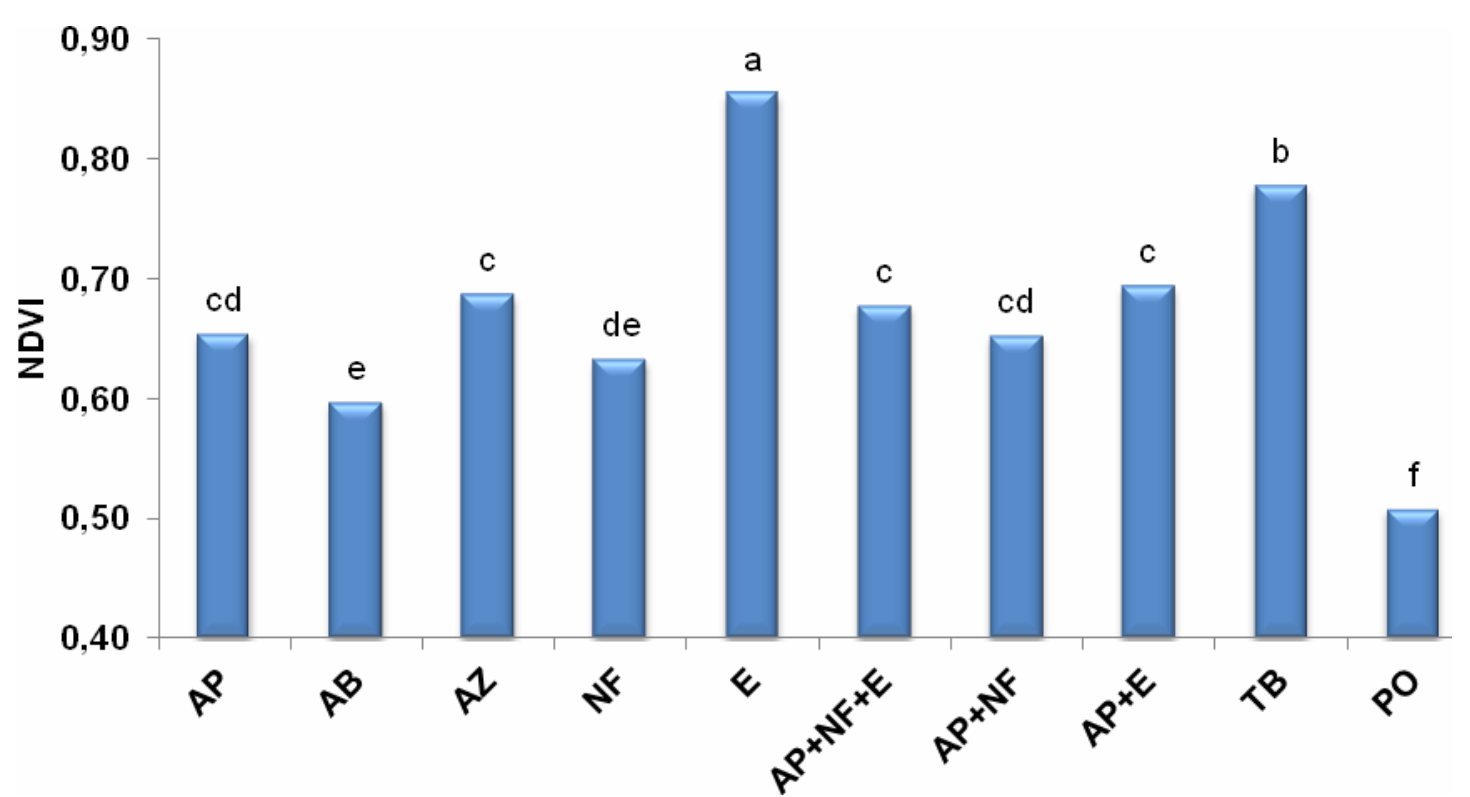

FIGURA 2. NDVI observado no momento da coleta em diferentes sistemas de plantas de cobertura do solo. Aveia preta (AP), aveia branca (AB), azevém (AZ), nabo forrageiro (NF), ervilhaca (E), tremoço branco (TB), vegetação espontânea ou pousio (PO). Médias seguidas de mesma letra, não diferem pelo teste Tukey a $5 \%$ probabilidade.

O índice de vegetação por diferença normalizada (NDVI) apresentou comportamento quadrático com a MMS produzida pelos dez sistemas de PCs analisados, obtendo-se coeficientes de determinação que variaram de 0,3158 a 0,7631 . De acordo com DEGHAID et al. (2001), estudando a correlação do NDVI com a cultura do amendoim, existe correlação positiva entre a refletância do dossel das plantas, expressa em valores de NDVI e a cobertura vegetal das culturas, encontrando um coeficiente de determinação de 0,8270 no estudo realizado.

Todos os tratamentos analisados apresentaram regressões polinomiais de segundo grau significativas, sendo que o tratamento $E$ apresentou forte correlação entre o NDVI e a MMS coletada $\left(R^{2}=0,7631\right)$, enquanto em $A P+E$ houve correlação moderada a forte $\left(R^{2}=0,6421\right)$, evidenciando que 0 índice testado é muito eficiente para estimar a quantidade de palhada produzida por estas PCs (Figura 3), dado que - NDVI apresenta associação significativa com a biomassa verde das plantas (PONZONI \& SHIMABUKURO, 2007; FONTANA et al., 2015), fundamental para mitigar o processo de degradação do solo e manter a qualidade do mesmo (COSTA et al., 2009; BEUTLER et al., 2014). 

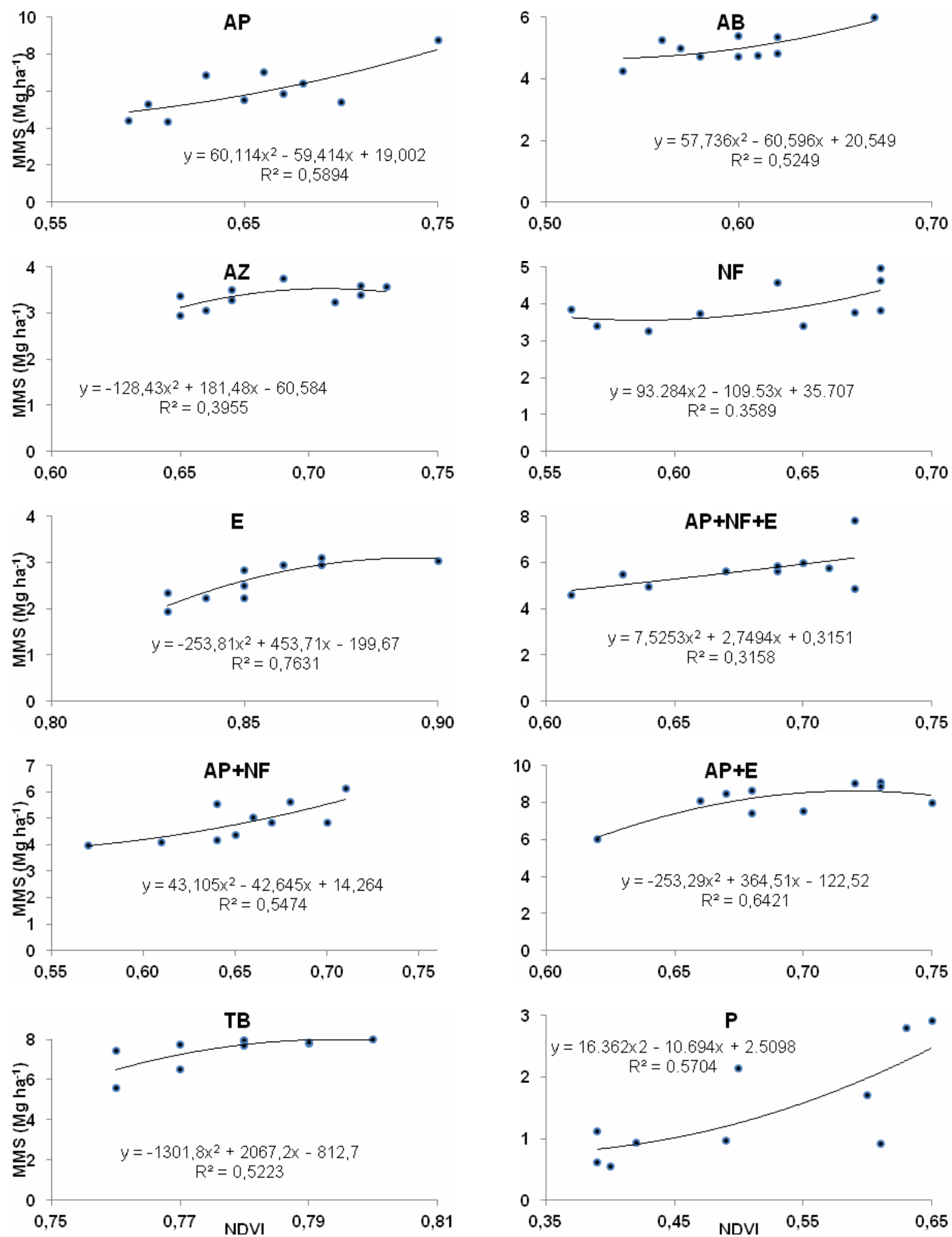

FIGURA 3. Análise de regressão entre a produção de fitomassa seca e NDVI em dez sistemas de plantas de cobertura do solo. MMS = Massa da matéria fresca. 
Os tratamentos $A P, A B, A P+N F$, TB e $P$ apresentaram correlação moderada entre o NDVI observado e a MMS produzida por estes sistemas, mostrando que esta ferramenta pode ser utilizada nestas PCs com certos ajustes ou até mesmo com novos testes em diferentes épocas de leituras. Destaca-se que no sensoriamento remoto, o uso de funções exponenciais é comum para expressar a relação da variável indicadora com o NDVI (LIU, 2006).

Já nos tratamentos $A Z$, NF e o consorciamento $A P+N F+E$, houve correlação fraca à moderada, variando de 0,3158 à 0,3955 , não sendo o NDVI indicado na determinação da MMS produzida por estes três sistemas. Devido ao SPD no sul do Brasil encerrar o ciclo das culturas de verão geralmente nos meses de março a abril, as PCs de inverno são semeadas na primeira quinzena de maio, sendo dessecadas em agosto para o plantio de milho, ou outubro para o plantio de soja, fazendo com que as coberturas AZ e NF já se encontrem em senescência ou até mesmo em florescimento, como foi o caso das mesmas, dificultando as leituras de NDVI, o que sugere a realização de leituras em diferentes épocas essencialmente nessas duas culturas, de modo a evitar que a presença de flores ou de o fim de ciclo possam interferir negativamente nos resultados, permitindo a utilização do NDVI para estimar a MMS produzida por estas.

Quanto ao consorciamento $\mathrm{AP}+\mathrm{NF}+\mathrm{E}$, provavelmente obteve uma correlação baixa pelo mesmo fato citado anteriormente em relação à comparação de diferentes PCs, não devendo ser analisadas de forma conjunta, possivelmente impossibilitando a utilização do NDVI nesse sistema de forma adequada, visto que as plantas são semeadas juntamente.

\section{CONCLUSÕES}

O NDVI foi capaz de determinar a fitomassa seca produzida de forma adequada ou satisfatória em sete dos dez sistemas analisados, sendo uma ferramenta que pode ser utilizada para estes fins nestes sistemas.

A presença de flores visíveis no dossel e a senescência natural atrapalharam a leitura de NDVI nos sistemas de plantas de cobertura do solo AZ e NF.

Não se recomenda a utilização deste índice para determinar a fitomassa produzida por consorciamento onde há a presença de três ou mais plantas, visto que o sensor não obteve bons resultados na estimativa da fitomassa produzida por diferentes plantas em conjunto.

\section{REFERÊNCIAS}

BEUTLER, A.N. Manejo do solo, palha residual e produtividade de arroz irrigado por inundação. Semina: Ciências Agrárias, Londrina, v.35, n.3, p. 1153-1162, 2014.

CARVALHO, W.P.; CARVALHO, G.J.; NETO, D.O.A.; TEIXEIRA, L.G.V. Desempenho agronômico de plantas de cobertura usadas na proteção do solo no período de pousio. Pesquisa Agropecuária Brasileira, Brasília, v.48, n.2, p.157166, 2013.

CARVALHO, A.M.; AMABILE, R.F. Plantas condicionadoras de solo: interações edafoclimáticas, uso e manejo. In: CARVALHO, A.M. de; AMABILE, R.F. (Ed.). Cerrado: adubação verde. Planaltina: Embrapa Cerrados, 2006. 369p. 
COSTA, A.; ALBUQUERQUE, J.A.; MAFRA, A.L.; SILVA, F.R. Propriedades físicas do solo em sistemas de manejo na integração agricultura-pecuária. Revista Brasileira de Ciência do Solo, Viçosa, MG, v.33, n.2, p.235-244, 2009.

CRUSIOL, L.G.T.; NEIVERTH, W.; RIO, A.; SIBALDELLI, R.N.R.; FERREIRA, L.C.; CARVALHO, J.F.; NEPUMUCENO, A.L.; NEUMAIER, N.; FARIAS, J.R.B. NDVI de estádios de desenvolvimento da soja BRS 284 em condições de campo. In: VIII Jornada Acadêmica da Embrapa Soja. Anais... Londrina: Embrapa Soja, 2013. p. 85-89.

DEGHAID, J.; ROSALEN, D.L.; ZERBATO, C.; FURLANI, C.E.A. Correlação entre o Índice de Vegetação por Diferença Normalizada (NDVI) e características agronômicas da cultura do amendoim. In: XLII Congresso Brasileiro de Engenharia CONBEA 2013. Anais... Campo Grande: SBEA, 2013. Não paginado.

DONEDA, A.; AITA, C.; GIACOMINI, S.J.; MIOLA, E.C.C.; GIACOMINI, D.A.; SCHIRMANN, J.; GONZATTO, R. Fitomassa e decomposição de resíduos de plantas de cobertura puras e consorciadas. Revista Brasileira de Ciência do Solo, v.36, p.1714-1723, 2012.

EMBRAPA, Empresa Brasileira de Pesquisa Agropecuária. Sistema brasileiro de classificação de solos. 3 ed. Brasília, 2013. 353 p.

FONTANA, D.C.; PINTO, D.G.; JUNGES, A.H.; BREMM, C. Inferências sobre o calendário agrícola a partir de perfis temporais de NDVI/MODIS. Bratantia, Campinas, no.ahead, p.000-000, 2015.

GROHS, D.S.; BREDEMEIER, C.; POLETTO, N.; MUNDSTOCK, C.M. Validação de modelo para predição do potencial produtivo de trigo com sensor óptico ativo. Pesquisa Agropecuária Brasileira, Brasília, v.46, n.4, p.446-449, abr. 2011.

LIU, W.T.H. Aplicações de sensoriamento remoto. Campo Grande: UNIDERP, 2006. $908 \mathrm{p}$.

MARCELO, A.V.; CORÁ, J.E.; FERNANDES, C.; MARTINS, M.R.; JORGE, R.F. Crop sequences in no-tillage system: Effects on soil fertility and soybean, maize and rice yield. Revista Brasileira de Ciência do Solo, v.33, n.2, p.417-428, 2009.

MENESES, P.R.; ALMEIDA T.. Brasília: UnB, 2012. 266 p.

OLLINGER, S.V. Sources of variability in canopy reflectance and the convergent properties of plants. New Phytologist, v.189, p.375-394, 2011.

PIMENTEL-GOMES, F. Curso de estatística experimental. São Paulo: Esalq, 1985. $467 \mathrm{p}$.

PONZONI, F.J.; SHIMABUKURO, Y.E. Sensoriamento remoto no estudo da vegetação. São José dos Campos: Parêntese. 2007. 127 p. 
RISSO, J.; RIZZI, R.; RUDORFF, B.F.T.; ADAMI, M.; SHUMABUKURO, Y.E.; FORMAGGIO, A.R.; EPIPHANIO, E.D.V. Índices de vegetação Modis aplicados na discriminação de áreas de soja. Pesquisa Agropecuária Brasileira, Brasília, v.47, n.9, p.1317-1326, 2012.

ROUSE, J.W.; HAAS, R.H.; SCHELL, J.A.; DEERING, D.W. Monitoring vegetation systems in the great plains with ERTS. In: III Earth Resources Technology Satellite Symposium. Anais... Washington: NASA, p.309-317.1973.

SIMÕES, C.R.; ROSSIELO, R.O.P.; GRACIOSA, M.G.; MACHADO, M.L.; SILVA, C.M. Imagens multiespectrais para avaliação de índice de área foliar e massa seca do capim 'Tifton 85', sob adubação nitrogenada. Ciência Rural, Santa Maria, v.45, n.4, p.697-703, 2015.

ZIECH, A.R.D.; CONCEIÇÃO, P.C.; GIRARDELLO, V.C.; HERBELE, C.T.; CASSOL, C.; GARMUS, T.G. Plantas de cobertura do solo na melhoria do Sistema Plantio Direto. Revista Plantio Direto, v.141, p.28-36, 2014. 